\title{
Sonication of retrieved implants improves sensitivity in the diagnosis of periprosthetic joint infection
}

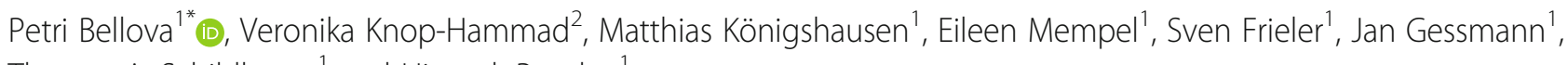
Thomas A. Schildhauer ${ }^{1}$ and Hinnerk Baecker ${ }^{1}$

\begin{abstract}
Background: Sonication is a valuable tool in the diagnosis of periprosthetic joint infections (PJI). However, conditions and definition criteria for PJ vary among studies. The aim of this study was to determine the diagnostic performance (i.e., specificity, sensitivity) of sonicate fluid culture (SFC) against periprosthetic tissue culture (PTC), when using European Bone and Joint Infection Society (EBJIS) criteria.

Methods: From March 2017 to April 2018, 257 implants were submitted for sonication. PJl was defined according to the EBJIS criteria as well as according to the International Consensus Meeting criteria of 2018 (ICM 2018). Only cases with at least one corresponding tissue sample were included. Samples were cultured using traditional microbiological plating techniques. Sensitivity and specificity were determined using two-by-two contingency tables. McNemar's test was used to compare proportions among paired samples. Subgroup analysis was performed dividing the cohort according to the site of PJl, previous antibiotic treatment, and time of manifestation. Prevalence of pathogens was determined for all patients as well as for specific subgroups.

Results: Among the 257 cases, 145 and 112 were defined as PJI and aseptic failure, respectively. When using the EBJIS criteria, the sensitivity of SFC and PTC was 69.0 and $62.8 \%$, respectively $(p=.04)$. Meanwhile, the specificity was 90.2 and $92.9 \%$, respectively $(p=.65$ ). When adopting ICM 2018 criteria, the sensitivity of SFC and PTC was 87.5 and $84.4 \%(p=.63)$ respectively, while the specificity was 85.1 and $92.5 \%(p=.05)$, respectively. The most commonly identified pathogens were coagulase-negative staphylococci (26\% overall), while $31 \%$ of PJI were culture-negative and $9 \%$ polymicrobial.

Conclusions: SFC exhibited significantly greater sensitivity versus PTC when using the EBJIS criteria. Nevertheless, the diagnosis of PJI remains a difficult challenge and different diagnostic tools are necessary to optimize the outcome.
\end{abstract}

Keywords: Periprosthetic joint infection, PJl, Hip, Knee, Sonication, Culture, Sensitivity, Specificity, Pathogen

\section{Background}

Periprosthetic joint infections (PJI) pose a major challenge in orthopedic surgery and traumatology. Every infection is a complication associated with multiple surgeries, prolonged hospitalization and significantly increased morbidity, while placing a major burden on the economy [1].

\footnotetext{
*Correspondence: petribellova@gmail.com

Hinnerk Baecker is a senior author

'Department of Orthopedic and Trauma Surgery, Surgical Clinic, BG

University Clinic Bergmannsheil Bochum, Bürkle-de-la-Camp Platz 1, 44789

Bochum, Germany

Full list of author information is available at the end of the article
}

Accurate diagnosis is crucial for the successful treatment of PJI. However, it may be difficult owing to the fact that there is no single test available with $100 \%$ sensitivity [2]. Thus, a combination of factors including clinical signs, laboratory results from peripheral blood and synovial fluid, microbiological culture and histological evaluation of periprosthetic tissue is necessary. The Musculoskeletal Infection Society (MSIS) and the Infectious Diseases Society have developed criteria to standardize the definition of PJI [3]. These criteria have been modified since their establishment $[4,5]$ leading to an increase in diagnostic confidence. Most recently,

(c) The Author(s). 2019 Open Access This article is distributed under the terms of the Creative Commons Attribution 4.0 International License (http://creativecommons.org/licenses/by/4.0/), which permits unrestricted use, distribution, and reproduction in any medium, provided you give appropriate credit to the original author(s) and the source, provide a link to the Creative Commons license, and indicate if changes were made. The Creative Commons Public Domain Dedication waiver (http://creativecommons.org/publicdomain/zero/1.0/) applies to the data made available in this article, unless otherwise stated. 
the ICM criteria were established as part of the 2018 International Consensus Meeting [5].

Previous criteria were designed for the diagnosis of definitive PJI. However, there is a considerable number of low-grade infections presenting with only subtle clinical and nonconfirmatory microbiological findings. These low-grade infections are often missed by common criteria. Based on this notion, a modified classification system for the diagnosis of PJI was proposed by Swiss Orthopaedics and the Swiss Society of Infectious Diseases (SOSSID) [6, 7]. In 2017, the SOSSID criteria were proposed by the European Bone and Joint Infection Society (EBJIS). These criteria use lower cutoff values for synovial fluid count and include sonication (Fig. 1).

Although tissue culturing has been the gold standard for the detection of causative pathogens, this approach is characterized by a high rate of false negatives $[8,9]$. The advantage of sonication lies in its capability to disrupt biofilms and thus, increase the number of microorganisms available for culture [10]. This allows for the administration of a more specific antibiotic treatment. Although several studies have shown improved sensitivity following the use of sonication, study conditions and definitions of PJI vary considerably.

The aim of this study was to determine the sensitivity and specificity of sonicate fluid culture (SFC) compared with periprosthetic tissue culture (PTC), using the EBJIS criteria as reference standard. In addition, for direct comparison, we defined PJI according to the current ICM criteria.

\section{Methods}

We retrospectively reviewed data of retrieved joint prostheses from March 2017 to April 2018. During this period, 257 implants were submitted for sonication. Data were obtained from the electronic records of the hospital. The local Ethical Review Committee approved this study (19-6603).

Inclusion criteria were the availability of sonicate fluid culture (SFC) for any prosthesis or prosthesis component between March 2017 and April 2018. Exclusion criteria were lack of corresponding tissue culture samples (at least 1 necessary for inclusion) and retrieval of any hardware other than prostheses or prostheses components.

For the definition of infection according to the EBJIS and ICM criteria, data including patient demographics (age, gender), surgery performed (type and date), clinical information (presence of a sinus tract or pus), microbiological and histopathological findings were obtained (Fig. 1a, b). Furthermore, information regarding preoperative administration of antibiotics, manifestation of the infection and duration of symptoms was collected. According to the respective definition criteria, cases were divided into PJI or aseptic failure (AF).

\section{PTC}

Intraoperative sampling of periprosthetic tissue was performed from the area macroscopically most suspicious of infection. Multiple samples were obtained in the vicinity of the implant. Cases lacking tissue samples were excluded from the analysis. In the microbiological laboratory, samples were prepared using forceps and scalpel under laminar air flow. Aliquots of the tissue were subsequently placed on different aerobic and anaerobic culture plates, and growth media (blood agar, chocolate agar, Schaedler agar, brain-heart infusion, Wilkens-Chalgren infusion). Culture was performed under human body temperature conditions $\left(37^{\circ} \mathrm{C}\right)$ for 14 days.

\section{SFC}

Explants were placed in sterile polypropylene containers that were opened in the operating room immediately prior to component explantation. In the laboratory, explants were immersed in Ampuwa (Fresenius Kabi Deutschland GmbH; Bad Homburg, Germany) solution and treated in an ultrasonic bath (BactoSonic; Bandelin, Berlin, Germany) for $60 \mathrm{~s}$ at $80 \% P=160 \mathrm{~W}$. Subsequently, $10 \mathrm{ml}$ of sonicate fluid were placed in aerobic and anaerobic blood culture bottles, followed by culture.

\section{Synovial fluid culture of preoperative joint aspiration (PJA)}

Synovial fluid was obtained under sterile conditions. The skin was disinfected thrice, and covered with sterile drapes. A skin incision was performed prior to aspiration. The retrieved joint fluid was subsequently divided into aliquots for microbiological and for cell counting analysis. White blood cell (WBC), polymorphonuclear neutrophil (PMN) and differential blood count were performed. The thresholds for WBC and PMN counts varied between the different criteria (Fig. 1).

\section{Periprosthetic membrane (PM)}

Histopathological examination of the PM was performed using the consensus classification established by Krenn and Morawietz [11]. A type 2 (septic failure) or type 3 (combined type).

PM was classified as infected.

As a retrospective study, not all diagnostic tools were deployed for each patient.

Subgroup analysis was performed dividing the cohort as follows:

1. Hip versus knee versus other

2. Previous antibiotic treatment versus no previous antibiotic treatment: any administration of antibiotics 14 days prior to surgery.

3. Early ( $<3$ months) versus delayed (3-24 months) versus late infection ( $>24$ months): time between introduction and removal of the implant. 


\begin{abstract}
A EBJIS criteria

one or more criteria required for diagnosis

Purulence around the prosthesis or sinus tract

Increased synovial fluid leukocyte count

Positive histopathology

Confirmatory microbial growth in synovial fluid, periprosthetic tissue, or sonication culture

Increased synovial fluid leukocyte count is indicated by a leukocyte count of $>2,000 / \mathrm{mL}$ or $>70 \%$ granulocytes; not interpretable within 6 weeks of surgery, in rheumatic joint disease, or after periprosthetic fracture or dislocation. Positive histopathology is defined as a mean of $>23$ granulocytes per $10 \mathrm{HPFs}$ (type II or type III), according to Krenn et al. (2006). Confirmatory microbial growth in periprosthetic tissue culture is considered positive if 1 specimen was positive in highly virulent organisms or 2 specimens show microbial growth of a low virulent pathogen. Sonication culture is considered positive if $>50$ colony-forming units $/ \mathrm{mL}$ of sonication fluid grow, or if $S$. aureus or anaerobes are
\end{abstract} detected irrespective of CFU

B major criteria

$\underline{\text { decision }}$

two positive cultures of the same organism

sinus tract with evidence of communication to the joint infected or visualisation of the prosthesis

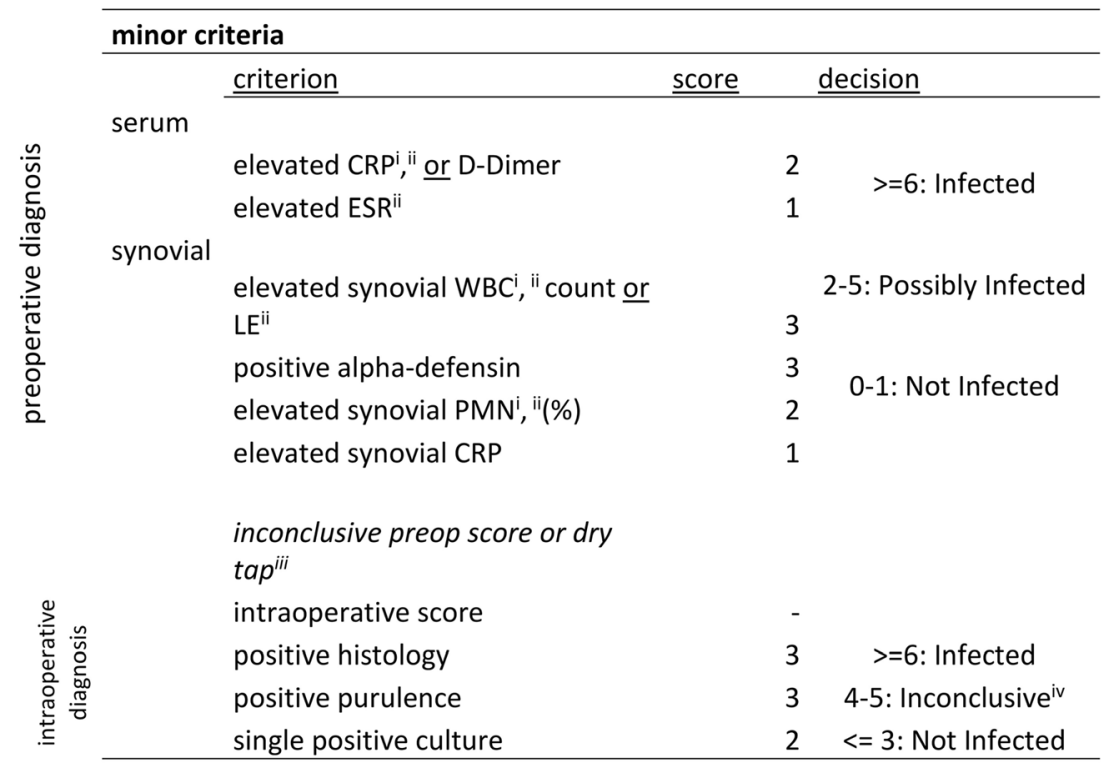

Fig. 1 a EBJIS criteria. b 2018 ICM criteria. ${ }^{i}$ thresholds

\begin{tabular}{|l|r|r|}
\hline marker & chronic (>90 days) & acute (<90 days) \\
\hline serum CRP & 1.0 & 10 \\
\hline synovial WBC count (cells/ $\mu \mathrm{l})$ & 3,000 & 10,000 \\
\hline synovial PMN $(\%)$ & 80 & 90 \\
\hline
\end{tabular}

sedimentation rate; WBC, white blood cell count; LE, leucocyte esterase; PMN, polymorphonuclear. ${ }^{\text {iii }}$ For patients with inconclusive minor criteria, operative criteria can be used to fulfill definition for PJI. ${ }^{\text {iii }}$ Further molecular diagnostics such as next generation sequencing should be considered

The incidence of microorganisms detected in SFC and PTC was recorded. Coagulase-negative staphylococci (CNS), anaerobes, streptococci and other microorganisms (including fungi among others) were labelled as pathogens of "low virulence". Methicillin-susceptible and methicillin-resistant Staphylococcus aureus (MSSA, MRSA), gram-negative rods and enterococci were labelled as pathogens of "high virulence". Sensitivity and specificity were calculated using two-by-two contingency tables. McNemar's test was used to compare 
proportions among paired samples. Statistical analysis was performed using the SPSS software (IBM Corporation; Armonk, NY, United States).

\section{Results}

A total of 257 cases of potentially infected prostheses were submitted for sonicate fluid analysis between March 2017 and April 2018. Using the EBJIS criteria as the reference standard, 145 and 112 cases were defined as infected and aseptic, respectively. According to the ICM criteria, there were 96 positive, 20 inconclusive and 141 negative cases. Baseline demographics between the two groups are shown in Table 1. The distribution of age, sex and affected joints were similar between cases of PJI and AF. Treatments differed between the two groups. Whereas the two-stage approach $(55.2 \%)$ was the predominant treatment type in PJIs, the one-stage approach (65.2\%) was the most prevalent treatment in $\mathrm{AF}$. In a total of 4 cases (1.6\%), salvage procedures were deployed. These included amputation, Girdlestone arthroplasty and local sinus care and suppressive antibiotics.

Among the 145 cases defined as PJI, 100 were accurately detected by SFC, whereas 45 were missed. Among the 112 cases defined as AF, 101 were accurately detected as negative by SFC, while eleven were falsely positive. This accounted for a sensitivity of $69.0 \%$, and a specificity of $90.2 \%$ for SFC. PTC was positive in 91 PJI and accurately negative in $104 \mathrm{AFs}$, leading to a sensitivity of $62.8 \%$ and a specificity of $92.9 \%$. The difference between SFC and PTC sensitivity was statistically significant $(p=0.04)$.

Table 1 Comparison of baseline demographics between PJI and aseptic failure group

\begin{tabular}{|c|c|c|c|c|}
\hline & & total $(n=257)$ & $\mathrm{PJI}^{1}(n=145)$ & $\mathrm{AF}^{2}(n=112)$ \\
\hline age, average & & & 70,0 & 69,5 \\
\hline sex male & & 84 (32.7\%) & $50(34.5 \%)$ & 34 (30.4\%) \\
\hline \multicolumn{5}{|l|}{ localisation } \\
\hline & shoulder & $21(8.2 \%)$ & 10 (6.9\%) & 11 (9.8\%) \\
\hline & knee & 89 (34.6\%) & 52 (35.9\%) & 37 (33.0\%) \\
\hline & hip & $143(55.6 \%)$ & 81 (55.9\%) & $62(55.4 \%)$ \\
\hline & other & $4(1.6 \%)$ & $2(1.4 \%)$ & $2(1.8 \%)$ \\
\hline \multirow[t]{2}{*}{ implant } & all & $146(56.8 \%)$ & 99 (68.3\%) & 47 (42.0\%) \\
\hline & component & $111(43.2 \%)$ & 46 (31.7\%) & 65 (58.0\%) \\
\hline \multicolumn{5}{|l|}{ surgery } \\
\hline & one stage & $105(40.9 \%)$ & 32 (22.1\%) & $73(65.2 \%)$ \\
\hline & two stage & 101 (39.3\%) & $80(55.2 \%)$ & 21 (18.7\%) \\
\hline & $D \& 1$ & 47 (18.3\%) & $30(20.7 \%)$ & $17(15.2 \%)$ \\
\hline & salvage & $4(1.6 \%)$ & $3(2.1 \%)$ & $1(0.9 \%)$ \\
\hline
\end{tabular}

${ }^{1} \mathrm{PJI}$, periprosthetic joint infection

${ }^{2} \mathrm{AF}$, aseptic failure
When using the ICM criteria, inconclusive results were excluded from the analysis of diagnostic validity. The sensitivity of SFC and PTC was $87.5 \%$ (84/96 cases) and $84.4 \%(81 / 96$ cases), respectively $(p=0.63)$. Moreover, the specificity was $85.1 \%(137 / 161$ cases $)$ and $92.5 \%(149 / 161)$, respectively $(p=0.05)$. Among 20 cases with inconclusive findings, six were SFC-positive and four were PTC positive.

In 127 out of 257 cases, both SFC and PTC were negative. In 130 out of 257, a pathogen was detected in either SFC or PTC or both of them. Among these 130 cases, 6 subgroups could be distinguished. The details are depicted in Table 2. The combined sensitivity and specificity of SFC and PTC was $76.6 \%(111 / 145)$ and $83.0 \%$ (93/112), respectively.

The subgroup analysis is shown in Table 3. Although sensitivity of SFC was higher than PTC in each subgroup, differences between SFC and PTC were not statistically significant. There was a trend towards higher sensitivity for SFC in late infections (59.5\% vs. $45.9 \% ; p=.06)$. Specificity did not differ among subgroups, neither.

PJA of synovial fluid was performed in 106 PJI and 73 AF. Among PJI, there were 41 positive PJA results (sensitivity: $38.7 \%$ ). 67 out of 73 PJA were accurately negative (specificity: 91.8\%).

Histopathological analysis of PM was available in 56 PJI and $13 \mathrm{AF}$. Among the PJI, 48 were indicative of an infection (sensitivity: 85.7\%). Samples for WBC and PMN were available in 78 out of 145 PJI and 56 out of $112 \mathrm{AF}$. Of the $78 \mathrm{PJI}, 26$ were excluded according to the limitations predetermined by the EBJIS criteria. Of the 52 valid samples, 26 were positive and negative, respectively (sensitivity: $50 \%$ ). Of the 56 joint aspirations

Table 2 results of sonicate fluid culture (SFC) and periprosthetic tissue culture (PTC) in periprosthetic joint infection (PJI) and aseptic failure (AF)

\begin{tabular}{llll}
\hline dignity & total & PJI & AF \\
\hline SFC+ ${ }^{1}$ PTC $^{2}$ & 35 & 24 & 11 \\
SFC+ PTC+ ${ }^{3}{ }^{4}{ }^{4}$ & 47 & 47 & 0 \\
SFC+ PTC+, D, ${ }^{5}$ add SFC ${ }^{6}$ & 5 & 5 & 0 \\
SFC+ PTC+ D, add PTC & 12 & 12 & 0 \\
SFC+ PTC+, D, diff & 12 & 12 & 0 \\
SFC- ${ }^{7}$ PTC- & 127 & 34 & 93 \\
SFC- PTC+ & 19 & 11 & 8 \\
& 257 & 145 & 112 \\
\hline
\end{tabular}

${ }^{1} \mathrm{SFC}+$, positive result ins sonicate fluid culture

${ }^{2}$ PTC-, negative result in periprosthetic tissue culture

${ }^{3} \mathrm{PTC}+$, positive result in periprosthetic tissue culture

${ }^{4} \mathrm{C}$, concordant

${ }^{5} \mathrm{D}$, discordant

${ }^{6}$ Add SFC, additional pathogen detected by sonicate fluid culture

${ }^{7}$ Add PTC, additional pathogen detected by periprosthetic tissue culture ${ }^{8}$ Diff, different findings

${ }^{9} \mathrm{SFC}-$, negative result in sonicate fluid culture 
Table 3 Subgroup analysis and respective diagnostic performance

\begin{tabular}{|c|c|c|c|c|c|c|c|}
\hline \multirow[t]{2}{*}{ subgroup } & & \multicolumn{3}{|c|}{ sensitivity (\%) } & \multicolumn{3}{|c|}{ specificity (\%) } \\
\hline & & $\overline{\text { SFC }}$ & PTC & $p^{1}$ & $\overline{S F C}$ & PTC & $p$ \\
\hline overall (EBJIS) & & 69.0 & 62.8 & .04 & 90.2 & 92.9 & .65 \\
\hline overall (ICM 2018) & & 87.5 & 84.4 & .63 & 85.1 & 92.5 & .05 \\
\hline \multicolumn{8}{|l|}{ joint } \\
\hline & hip $(n=143)$ & 70.4 & 63.0 & .24 & 91.9 & 90.3 & 1.00 \\
\hline & knee $(n=89)$ & 65.4 & 53.8 & .15 & 91.9 & 94.6 & 1.00 \\
\hline & other $(n=25)$ & 75.0 & 66,7 & 1.00 & 76.9 & 100.0 & $p^{2}$ \\
\hline \multicolumn{8}{|c|}{ previous $\mathrm{AB}^{3}$ treatment } \\
\hline & antibiotics $(n=63)$ & 72.3 & 57.4 & .12 & 100.0 & 100.0 & / \\
\hline & no antibiotics $(n=194)$ & 67.3 & 61.2 & .36 & 88.5 & 91.7 & .65 \\
\hline \multicolumn{8}{|c|}{ introduction to removal } \\
\hline & early $(n=104)$ & 76.5 & 67.6 & .36 & 94.4 & 91.7 & 1.00 \\
\hline & delayed $(n=72)$ & 65.0 & 60.0 & .75 & 78.1 & 93.7 & .18 \\
\hline & late $(n=81)$ & 59.5 & 45.9 & .06 & 95.5 & 93.2 & 1.00 \\
\hline
\end{tabular}

${ }^{1}$ McNemar's test of paired proportions. $P$ values in italics. A $p$ value $<.05$ indicates statistical significance. Slgnificant values are displayed in fat.

$2 /$, not applicable

${ }^{3} \mathrm{AB}$, antibiotic

in AF, 24 were excluded. The remaining 32 samples were all negative (specificity: 100\%; Fig. 2).

The absolute distribution is shown in Table 4 whereas the relative distribution of pathogens detected by SFC is illustrated in Fig. 3. The culture-negative rate was 31.0\% (45/145) and $40.0 \%$ (58/145) for SFC and PTC, respectively. A polymicrobial result was obtained in $13(9.0 \%)$ and $20(13.8 \%)$ PII in SFC and PTC, respectively. In early PJI, $48.9 \%(22 / 45)$ and $51.1 \%(23 / 45)$ of pathogens detected through SFC were of high and low virulence, respectively. In delayed and late infections combined, $35.7 \%$ (15/42) and 64.3\% (27/42) of pathogens detected through SFC were of high and low virulence, respectively. Most notably, 7.8\% (6/77) of delayed or late infections included the detection of anaerobes, with Cutibacterium acnes as the most common representative (5/6). The corresponding detection rate for PTC was 2.6\% (2/77).

\section{Discussion}

Diagnosis of PJI is challenging owing to the existing biofilm around implants [12]. Pathogens embedded in a biofilm are enclosed in a polymeric matrix and have altered their phenotype into an extremely resilient form of life, protecting them from antimicrobial and host immune responses [13]. Biofilm bacteria exhibit a markedly higher resistance to antimicrobial killing compared with planktonic bacteria [1].

Sonication was popularized as a diagnostic tool for PJI by Trampuz et al. [14]. During the process, adherent pathogens are dislodged from the surface of the implant through low-frequency ultrasound, preserving their viability [15]. Various studies have shown an improved sensitivity following the use of sonication compared with conventional tissue culture. In a meta-analysis (16 studies) performed by Liu et al. [12], sonication yielded a pooled sensitivity and specificity of $79 \%$ (95\% confidence interval $[\mathrm{CI}]: .76-.81$ ) and 95\% (95\% CI: .94-.96), respectively. The results were similar in a meta-analysis (12 studies) conducted by Zhai et al. [16], showing a pooled sensitivity and specificity of $80 \% \quad(95 \%$ CI: $.74-.84$ ) and 95\% (95\% CI: .90-.98), respectively. Furthermore, a meta-analysis of four studies investigating sonicate fluid in blood culture bottles displayed a sensitivity and specificity of $85 \%$ (95\% CI: .77-.91) and 86\% (95\% CI .81 to .91), respectively [17]. However, the reference standards for the diagnosis of PJI differed among the studies included in the meta-analyses.

In our study, SFC showed low sensitivity $(69.0 \%)$ compared with previous data regarding sonication $[12,16]$. Overall, we detected 45 false negative PJI. However, PJI was defined according to the EBJIS criteria, as having a low threshold for the detection of an infection.

When adopting the ICM criteria, the sensitivity and specificity of SFC were comparable with those reported in recent meta-analyses (87.5 and $93.5 \%$, respectively), after the exclusion of inconclusive findings. The discrepancy between the EBJIS criteria and more commonly used criteria (i.e., MSIS) has been reproduced in a recent study performed by Renz et al. [18] which sought to determine the diagnostic value of the alpha defensin lateral flow test using different classification systems. The sensitivity of this test was $84 \%$ by MSIS criteria but only $54 \%$ by EBJIS criteria.

We found a significantly higher sensitivity of SFC compared with PTC $(69.0 \%$ vs. $62.8 \%$, respectively; $p=$ $0.04)$. However, the specificity was similar $(90.2 \%$ vs. 


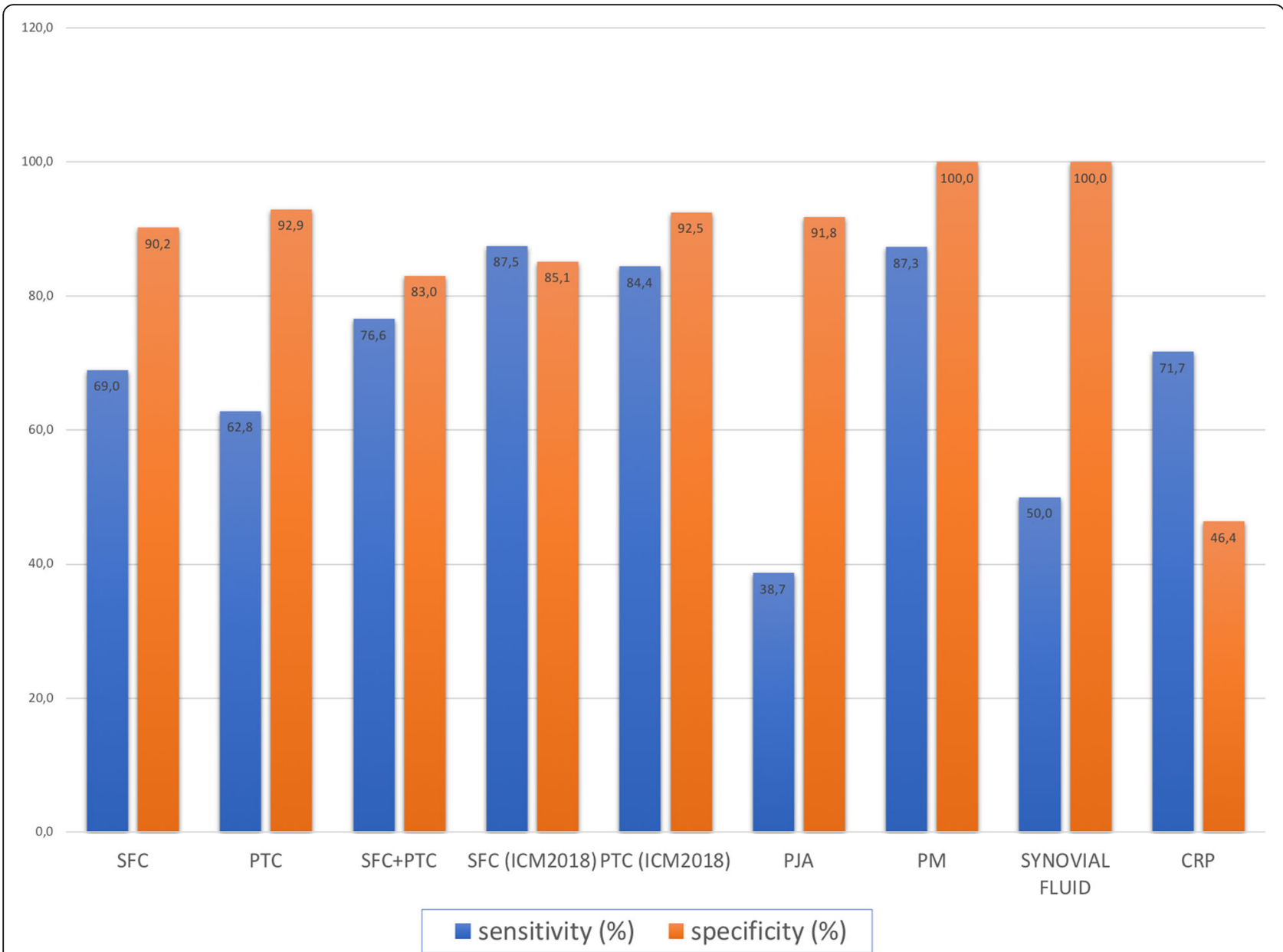

Fig. 2 diagnostic performance (sensitivity, specificity) of diagnostic tools deployedVertical axis represents the value of diagnostic performance in $\%$. On the horizontal axis, the respective tools are depicted. Abbreviations are explained in the text. Blue columns represent sensitivity while orange columns represent specificity

Table 4 overall distribution of microorganisms and in early, delayed, late infection as detected by sonicate fluid culture (SFC) and periprosthetic tissue culture (PTC)

\begin{tabular}{|c|c|c|c|c|c|c|c|c|}
\hline & early & & delay & & late ( & & total & \\
\hline & SFC & PTC & SFC & PTC & SFC & PTC & $\overline{S F C}$ & PTC \\
\hline $\mathrm{CNS}^{1}$ & 18 & 15 & 14 & 7 & 6 & 8 & 38 & 30 \\
\hline$M S S A^{2}$ & 9 & 6 & 1 & 1 & 4 & 5 & 14 & 12 \\
\hline$M R S A^{3}$ & 2 & 1 & 2 & 2 & 1 & 1 & 5 & 4 \\
\hline $\operatorname{gram}(-)$ rods $^{4}$ & 9 & 6 & 2 & 2 & 2 & 1 & 13 & 9 \\
\hline anaerobes & 1 & 2 & 4 & 2 & 2 & 0 & 7 & 4 \\
\hline enterococci & 2 & 0 & 2 & 1 & 1 & 0 & 5 & 1 \\
\hline streptococci & 1 & 0 & 0 & 2 & 0 & 0 & 1 & 2 \\
\hline other & 3 & 3 & 0 & 1 & 1 & 1 & 4 & 5 \\
\hline polymicrobial & 7 & 13 & 1 & 6 & 5 & 1 & 13 & 20 \\
\hline no growth & 16 & 22 & 14 & 16 & 15 & 20 & 45 & 58 \\
\hline
\end{tabular}

${ }^{1}$ CNS coagulase-negative staphylococci

${ }^{2}$ MSSA methicillin succeptible Staphylococcus aureus

${ }^{3}$ MRSA methicillin resistant staphylococcus aures

${ }^{4}$ Gram (-) rods, gram-negative rods 


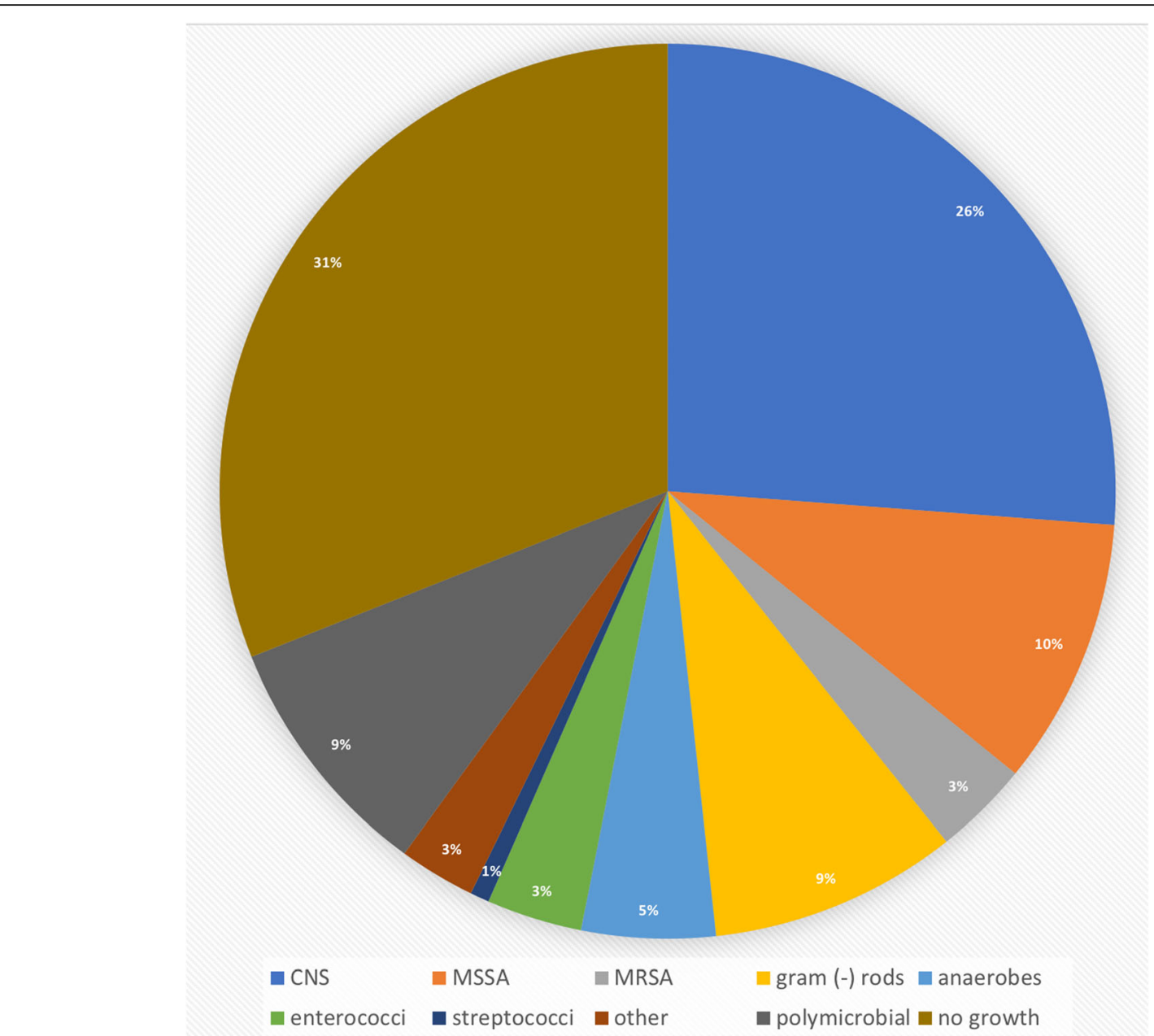

Fig. 3 Relative prevalence of pathogens as detected by sonicate fluid culture (SFC)Abbreviations are explained in the text and Table 4. The legend within the figure explains which color represents which entity

$92.9 \%$, respectively; $p=.65$ ). Our results were in concordance with those of recent studies, that have mostly observed a superior sensitivity of SFC compared with PTC $[10,19,20]$. Two recent studies showed that the sensitivity of SFC was lower than that of PTC [21, 22]. The discrepancy between studies can be attributed to the varying study conditions and definitions of PJI. In particular, the colony-forming unit (CFU) value required to define a positive SFC result differed greatly among studies.

Subgroup analysis showed a superior sensitivity of SFC throughout all subgroups. However, there were no significant differences between SFC and PTC among subgroups. There was a somewhat higher sensitivity for SFC of hip implants when compared with knee implants (hip: $70.4 \%$ vs. knee: $65.4 \%$ ). A possible explanation may be the more frequent use of antibiotic-loaded cement in knee arthroplasty, which may disturb the structure of the biofilm [23]. However, SFC of knee implants might provide a greater advantage against PTC when compared with SFC of hip implants (knee: $p=.15$ vs hip: $p=.24$ ). Previous administration of antibiotics did not influence the sensitivity of SFC, confirming the findings of comparable studies $[24,25]$. However, there was a trend towards higher sensitivity of SFC against PTC $(p=.12)$ in the antibiotics groups as opposed to when antibiotics were not administered $(p=.36)$. It has to be noted, however, that the antibiotic group was characterized by a relatively small sample size $(n=63)$.

We found both SFC and PTC sensitivity to drop consecutively with delayed and late infections. Low sensitivity in late infections might be a result of EBJIS criteria. A high amount of cases in this group would have been classified as aseptic under alternative definition criteria. Furthermore, we found a strong trend towards higher sensitivity of SFC against PTC in the "late infections" group $(59.5 \%$ vs. $45.9 \%$; $p=0.06)$ which was in concordance with a recent study by Puig- Verdié et al. [26]. Apparently, in early infection, a majority of microorganisms are expected to not have formed biofilms yet, leading to 
high detection rates both in SFC and in PTC. In late infection, meanwhile, a biofilm is expected to have formed by most microorganisms and sonication should provide an advantage against conventional culturing.

The prevalence of pathogens was in concordance with current literature. CNS, as in our study (26\%), were by far the most common pathogens in various studies across the board [6, 10, 27-29]. The relatively high prevalence of gram-negative rods (9\%), especially in early infection $(9 / 81,11 \%)$, is also a finding supported by literature [29]. Moreover, the detection of six anaerobes in 77 cases $(7.8 \%)$ of delayed and late infections is a finding concordant with literature [28]. Interestingly, out of the six cases, four times anaerobes were detected by SFC alone supporting the notion that these microorganisms are especially susceptible to SFC and may be missed by PTC [15]. With EBJIS criteria, any detection of anaerobes in SFC is a PJI, irrespective of CFU.

We found a comparably high prevalence of culturenegative PJI (31\%). In a recent meta-analysis, Reisener et al. [30] reported that the incidence of culture-negative PJI ranged from 7 to $42 \%$. As expected, we found that highly virulent pathogens were more prevalent in early infection than in delayed or late infection, confirming the findings of previous studies [26, 31]. The SFC and PTC results were similar, however, culture-negative results were more frequent in PTC.

The present study has several limitations. Firstly, it is limited by its retrospective nature. Not all elements of the EBJIS PJI criteria were available for each patient. PJA was available in 73 and $65 \%$ of PJI and AF cases. WBC/ PMN was available in 54 and $50 \%$ of PJI and AF cases, respectively. Moreover, PM was available in only 38 and $13 \%$ of PJI and AF cases, respectively. An explanation for the lack of data may be the new introduction of standardized principles in our clinic.

Diagnosis according to the ICM criteria may have been biased owing to missing data. The rate of erythrocyte sedimentation among serum markers, as well as leukocyte esterase, alpha defensin and synovial Creactive protein among synovial markers, were not part of the diagnostic routine. The resulting score contained only points received from available tools.

Besides cases defined as "infected" and "not infected", the new ICM criteria allow for the detection of "inconclusive" results in patients with a score of 4 or 5 . In our study, 20 patients were defined as such. These patients present a real diagnostic challenge and might benefit from molecular diagnostic testing such as next generation sequencing which- of course- was not at our disposal.

Colony counting was not performed with sonication. Especially in SFC(+)PTC(-) cases, measurement of CFU is of high importance in distinguishing between infection and contamination. According to the EBJIS criteria, a CFU count of $>50 \mathrm{CFU} / \mathrm{ml}$ is indicative of an infection. In this study, this conflict was solved by interpreting SFC as an additional PTC, and subsequently applying the respective rules. Our method of sonication does not include the step of vortexing. In a subgroup metaanalysis [16], cases that included vortexing within the process of sonication exhibited higher sensitivity (79\% vs. $78 \%$, respectively) and higher specificity ( $96 \%$ vs. $85 \%)$ versus those that did not.

The strength of this study lies in its relatively high sample size $(n=257)$, which allowed for a reliable comparison between SFC and PTC, and yielded large subgroups for further analyses. Furthermore, definition of PJI and aseptic failure was performed in a very meticulous manner, including all aspects of the respective definition criteria. To our knowledge, this is the first study investigating sonication using the EBJIS criteria as a reference standard for PJI, while simultaneously incorporating the most recent ICM criteria.

\section{Conclusions}

We were able to confirm that SFC has a better sensitivity than PTC based on EBJIS but not on ICM criteria. The specificity, meanwhile, was similar for the two based on both reference standards. According to these findings, improvement of the diagnostic accuracy of sonication is still necessary and the diagnosis of PJI continues to require a combination of diagnostic tools.

\section{Abbreviations}

AF: Aseptic failure(s); Cl: Confidence intervall; CNS: Coagulase-negative staphylococci; CRP: C-reactive protein; EBJIS: European Bone and Joint Society; ESR: Erythrocyte sedimentation rate; ICM: International Consensus Meeting (criteria); LE: Leucocyte esterase; MRSA: Methicillin-resistant Staphylococcus aureus; MSIS: Musculoskeletal Infection Society; MSSA: Methicillin-susceptible Staphylococcus aureus; PJA: Preoperative joint aspiration (for synovial fluid culture); PJl: Periprosthetic joint infection(s); PM: Periprosthetic membrane; PMN: Polymorphonuclear neutrophil (percentage); PTC: Periprosthetic tissue culture; SFC: Sonicate fluid culture; SOSSID: Swiss Orthopaedics and the Swiss Society of Infectious Diseases; WBC: White blood cell

\section{Acknowledgments}

The manuscript underwent proof-reading with Charlesworth Author Services. The content of the manuscript has not been published and is not under consideration for publication elsewhere.

\section{Authors' contributions}

PB, EM and SF retrieved, analyzed and interpreted the patient data. PB and VK were responsible for the analysis of microbiological data. PB, MK as well as JG contributed to the statistical analysis of data. HB and TS, along with PB contributed to the writing and revising of the manuscript. All authors read and approved the final manuscript.

\section{Funding}

We acknowledge support by the DFG Open Access Publication Funds of the Ruhr-Universität Bochum. The funding body did neither play a role in the design of the study, nor in the collection, analysis and interpretation of data, nor in writing the manuscript. 


\section{Availability of data and materials}

The datasets used and/or analysed during the current study are available from the corresponding author on reasonable request.

\section{Ethics approval and consent to participate}

This study was approved by the Ethical Review Board of Ruhr-University Bochum (18-6603-BR).

\section{Consent for publication}

not applicable.

\section{Competing interests}

The authors declare that they have no competing interests.

\section{Author details}

'Department of Orthopedic and Trauma Surgery, Surgical Clinic, BG University Clinic Bergmannsheil Bochum, Bürkle-de-la-Camp Platz 1, 44789 Bochum, Germany. ${ }^{2}$ Department of Microbiology, BG University Clinic Bergmannsheil Bochum, Bochum, Germany.

Received: 29 August 2019 Accepted: 16 December 2019 Published online: 26 December 2019

\section{References}

1. Zimmerli W, Trampuz A, Ochsner PE. Prosthetic-joint infections. N Engl J Med. 2004;351(16):1645-54

2. Fernández-Sampedro M, Fariñas-Alvarez C, Garces-Zarzalejo C, et al. Accuracy of different diagnostic tests for early, delayed and late prosthetic joint infection. BMC Infect Dis. 2017;17(1):592.

3. Parvizi J, Zmistowski B, Berbari EF, et al. New definition for periprosthetic joint infection. From the workgroup of the musculoskeletal infection society. Clin Orthop Relat Res. 2011:469(11):2992-4.

4. Parvizi J, Gehrke T, Chen AF. Proceedings of the international consensus on Periprosthetic joint infection. Bone Joint J. 2013;95-B(11):1450-2.

5. Parvizi J, Tan TL, Goswami K, et al. The 2018 definition of Periprosthetic hip and knee infection. An evidence-based and validated criteria. J Arthroplast. 2018;93(5):1309-14.

6. Corvec S, Portillo ME, Pasticci BM, Borens O, Trampuz A. Epidemiology and new developments in the diagnosis of prosthetic joint infection. Int J Artif Organs. 2012;35(10):923-34.

7. Ochsner PE, Swiss Orthopaedics, Swiss Society for Infectious Diseases. Infections of the musculoskeletal system - basic principles, prevention, diagnosis and treatment. 1st ed. Grandveaux: Swiss Orthopaedics; 2014.

8. Spangehl MJ, Masri BA, O'Connell JX, Duncan CP. Prospective analysis of preoperative and intraoperative investigations for the diagnosis of infection at the sites of two hundred and two revision total hip arthroplasties. J Bone Joint Surg Am. 1999:81(5):672-83.

9. Baré J, MacDonald SJ, Bourne RB. Preoperative evaluations in revision total knee arthroplasty. Clin Orthop Relat Res. 2006:446:40-4.

10. Rothenberg AC, Wilson AE, Hayes JP, O'Malley MJ, Klatt BA. Sonication of Arthroplasty implants improves accuracy of Periprosthetic joint infection cultures. Clin Orthop Relat Res. 2017:475(7):1827-36.

11. Morawietz L, Classen RA, Schröder JH, et al. Proposal for a histopathological consensus classification of the periprosthetic interface membrane. J Clin Pathol. 2006;59(6):591-7

12. Liu H, Zhang Y, Li L, Zou HC. The application of sonication in diagnosis of periprosthetic joint infection. Eur J Clin Microbiol Infect Dis. 2017;36(1):1-9.

13. Costerton JW, Montanaro L, Arciola CR. Biofilm in implant infections. Its production and regulation. Int J Artif Organs. 2005;28(11):1062-8.

14. Trampuz A, Piper KE, Jacobson MJ, et al. Sonication of removed hip and knee prostheses for diagnosis of infection. N Engl J Med. 2007; 357(7):654-63.

15. Renz N, Cabric S, Janz V, Trampuz A. Sonication in the diagnosis of periprosthetic infections: significance and practical implementation. Orthopade. 2015;44(12):942-5.

16. Zhai Z, Li H, Qin A, et al. Meta-analysis of sonication fluid samples from prosthetic components for diagnosis of infection after total joint arthroplasty. J Clin Microbiol. 2014;52(5):1730-6.

17. Li C, Renz N, Trampuz A. Management of Periprosthetic Joint Infection. Hip Pelvis. 2018;30(3):138-46.
18. Renz N, Yermak K, Perka C, Trampuz A. Alpha Defensin lateral flow test for diagnosis of Periprosthetic joint infection. Not a screening but a confirmatory test. J Bone Joint Surg Am. 2018;100(9):742-50.

19. Prieto-Borja $L$, Auñón Á, Blanco $A$, et al. Evaluation of the use of sonication of retrieved implants for the diagnosis of prosthetic joint infection in a routine setting. Eur J Clin Microbiol Infect Dis. 2018;37(4):715-22.

20. Ueda N, Oe K, Nakamura T, et al. Sonication of extracted implants improves microbial detection in patients with orthopedic implant-associated infections. J Arthroplast. 2019:34(6):1189-96.

21. van Diek FM, Albers CGM, van Hooff ML, Meis JF, Goosen JHM. Low sensitivity of implant sonication when screening for infection in revision surgery. Acta Orthop. 2017;88(3):294-9.

22. Dudareva M, Barrett L, Figtree $M$, et al. Sonication versus Tissue Sampling for Diagnosis of Prosthetic Joint and Other Orthopedic Device-Related Infections. J Clin Microbiol. 2018:56(12):00688-18(1-12).

23. Tani S, Lepetsos P, Stylianakis A, et al. Superiority of the sonication method against conventional periprosthetic tissue cultures for diagnosis of prosthetic joint infections. Eur J Orthop Surg Traumatol. 2018;28(1):51-7.

24. Sampedro MF, Huddleston PM, Piper KE, et al. A biofilm approach to detect bacteria on removed spinal implants. Spine. 2010;35(12):1218-24.

25. Scorzolini $L$, Lichtner $M$, lannetta $M$, et al. Sonication technique improves microbiological diagnosis in patients treated with antibiotics before surgery for prosthetic joint infections. New Microbiol. 2014;37(3):321-811.

26. Puig-Verdie L, Alentorn-Geli E, Gonzalez-Cuevas A, et al. Implant sonication increases the diagnostic accuracy of infection in patients with delayed, but not early, orthopaedic implant failure. Bone Joint J. 2013:95-B(2):244-9.

27. Del Pozo JL, Patel R. Clinical practice. Infection associated with prosthetic joints. N Engl J Med. 2009;361(8):787-94.

28. Drago $L$, de Vecchi $E$, Bortolin $M$, et al. Epidemiology and antibiotic resistance of late prosthetic knee and hip infections. J Arthroplast. 2017; 32(8):2496-500.

29. Zeller V, Kerroumi Y, Meyssonnier V, et al. Analysis of postoperative and hematogenous prosthetic joint-infection microbiological patterns in a large cohort. J Inf Secur. 2018;76(4):328-34

30. Reisener M, Perka C. Do culture-negative Periprosthetic joint infections have a worse outcome than culture-positive Periprosthetic joint infections? A Systematic Review and Meta-Analysis. Biomed Res Int. 2018.

31. Tande AJ, Patel R. Prosthetic joint infection. Clin Microbiol Rev. 2018; 6278012:1-12

\section{Publisher's Note}

Springer Nature remains neutral with regard to jurisdictional claims in published maps and institutional affiliations.

Ready to submit your research? Choose BMC and benefit from:

- fast, convenient online submission

- thorough peer review by experienced researchers in your field

- rapid publication on acceptance

- support for research data, including large and complex data types

- gold Open Access which fosters wider collaboration and increased citations

- maximum visibility for your research: over $100 \mathrm{M}$ website views per year

At BMC, research is always in progress.

Learn more biomedcentral.com/submissions 